\title{
Gartenraum und Zeremoniell
}

\author{
Marcus Köhler
}

$\mathrm{D}$ ie Rückführung des Großen Gartens in Hannover-Herrenhausen in seinen barocken Zustand im Jahr 1937 kann man als Akt schöpferischer Denkmalpflege bezeichnen. Er ist somit bis heute auch ein Zeichen der damaligen Anschauung über das Barock. Entsprechend frei wurde die Geschichte interpretiert: Im Umkreis der Wiederherstellung entstand, auch durch Wissenschaftler wie Udo von Alvensleben gefördert, die Vorstellung eines »festlichen Herrenhausens", das maßgeblich von der Kurfürstin Sophie (1630-1714) geprägt wurde. ${ }^{1}$ Dieser Blick hat die bisherige Forschung eher belastet als gefördert, da er weitgehend nicht den historischen Tatsachen entspricht. ${ }^{2}$ Rosemarie Wallbrecht kann als eine der ersten gelten, die mittels ihrer hannoverschen Theatergeschichte den festlichen Höhepunkt des Sommersitzes erst zwischen 1730-1750 sah. Auch zeigte sie, dass die »Festlichkeit» nicht durchgängig war, sondern sich auf einzelne Aktionen beschränkte. ${ }^{3}$ Ungeachtet dessen bestimmt jedoch noch immer die Vorstellung einer durchgängigen Festlichkeit des Barockgartens die Forschung, wobei das Motiv des Festes zumeist mit der Sphäre des Höfischen gleichgesetzt wird. Die Titelwahl einer Ausstellung im Jahr 2000 »Gartenfeste. Das Fest im Garten - Gartenmotive im Fest « führte konsequenterweise zu einer undifferenzierten Darstellung, hinter der sich lediglich die höfische Rezeption zeitgenössischer Gartenkultur verbarg. ${ }^{4}$ Festlichkeit und Zeremoniell spielen dabei nur am Rande eine Rolle. Da sich im Königlichen Hausarchiv Hannover die sogenannten Zeremonialbücher des Hofes erhalten haben, die in etwa die Zeit von 1680 bis 1830 abdecken, soll an dieser Stelle untersucht werden, wie sich Festlichkeit, Außenraum und Zeremoniell tatsächlich zueinander verhielten. ${ }^{5}$

Folgt man den Aufzeichnungen, so fand in dem kleinen und unspektakulären Garten in Herrenhausen zunächst kein Hofzeremoniell statt. Erst nach Verleihung der neunten Kur im Jahr 1692 baute man ihn parallel zum Garten des dadurch übervorteilten Vetters Herzog Anton Ulrich von Braunschweig in Salzdahlum aus, musste jedoch gleichzeitig sehen, dass durch die Zahlung von Subsidiengeldern die ruinierten Kassen kaum Entfaltungsmöglichkeiten boten. Lediglich der Vorhof spielte zunächst eine Rolle beim Empfang diverser Botschafter. ${ }^{6} \mathrm{Im}$ Sommer wurden sie mit Kutschen aus der Stadt nach Herrenhausen zur Audienz gebracht. Dabei fuhr man zwei Mal durch die zwei Kilometer lange Allee, die unmittelbar nach Erlangung der Kurfürstenwürde mit schnell wachsenden Pappeln angepflanzt wurde, um als "Prozessionsstrecke» rasch einen majestätischen Eindruck zu vermitteln. ${ }^{7}$

Innerhalb des Schlossbezirks verzeichnet das Zeremonialbuch akribisch, ob die Kutschen vor oder unter dem Tor oder gar erst an der Treppe hielten. Je höher der Rang, desto weniger brauchten die Gäste zu Fuß zu gehen. Bei Anwesenheit hoher Fürsten wurde der Hof vor das Schloss ins Freie befohlen. In besonderen Fällen holte man sogar Gäste ein, wie etwa 1681 die verwitwete Tante Sophie Amalie von Dänemark, die eine Meile vor Hannover in einem "großen Gezelt» vom versammelten Hof in Gala in 
Empfang genommen wurde. ${ }^{8}$ Schwieriger war es, 1697 dem inkognito reisenden $\mathrm{Za}$ ren Peter in Coppenbrügge aufzuwarten. ${ }^{9}$ Protokollarische und organisatorische Verwicklungen traten auf. Grund dafür war, dass außerhalb der Residenz, nämlich im bescheidenen Amtshaus zu Coppenbrügge, in dem die Szene stattfand, fernab jeglicher Raumfolgen und Festsäle ein Zeremoniell schwer oder gar nicht mehr zu erfüllen war. Über eine vergleichbare skurrile Begebenheit weiß auch der hannoversche Stadtchronist Redecke im Jahr 1716 zu berichten:

»Am 5. Juni kam der König in Preußen, Friedrich [Wilhelm I.], mit dreyen Cavalliers, auf einem offenen Wagen, an dem Posthause außer dem Steinthor an, lief auf Nicolai Kirchhof und ließ sich ein Glas Bier bringen, welches er unter den Lindenbäumen austrank. Indessen wechselte man die Pferde, und er stieg wieder zu Wagen. Als aber eben der nunmehrige Bischof zu Osnabrück, Herzog Ernst August, des Königs Georg des ersten Bruder, neben andern Großen, durch den Geheimen Rath und Großvoigt
Joachim Heinrich Freyherrn von Bülow, auf dessen an dem Heerwege bey gedachtem Kirchhofe belegenen Garten tractiret wurde, und eben da die Mahlzeit anging, der König in Preußen vorbey jagen wollte, fiel die gantze hohe und vornehme Gesellschaft seine Pferde und Wagen an und baten so viel, daß er sich auch zur Tafel setzte und gut speisete. ${ }^{10}$

Aktionen außerhalb der Schlösser schufen ein protokollarisches Vakuum. Um nicht die skurrile Begegnung von Coppenbrügge zu wiederholen, ging man in Hannover dazu über, aus diplomatischem Kalkül oder Freundlichkeit auch im Freien eine Art des Zeremoniells anzubieten. Dem Gast stand es frei, sich darauf einzulassen oder dies abzulehnen, was vor allem bei Abwesenheit des Monarchen von Bedeutung war. So verzeichnet das Zeremonialbuch am 18. August 1768 anlässlich einer Durchreise des preußischen Königs Friedrich II., dass er vor dem Rühlingschen Garten

"wegen alda versamleten Hofes zwahr ausgestiegen doch nicht ins Haus zur Ein-

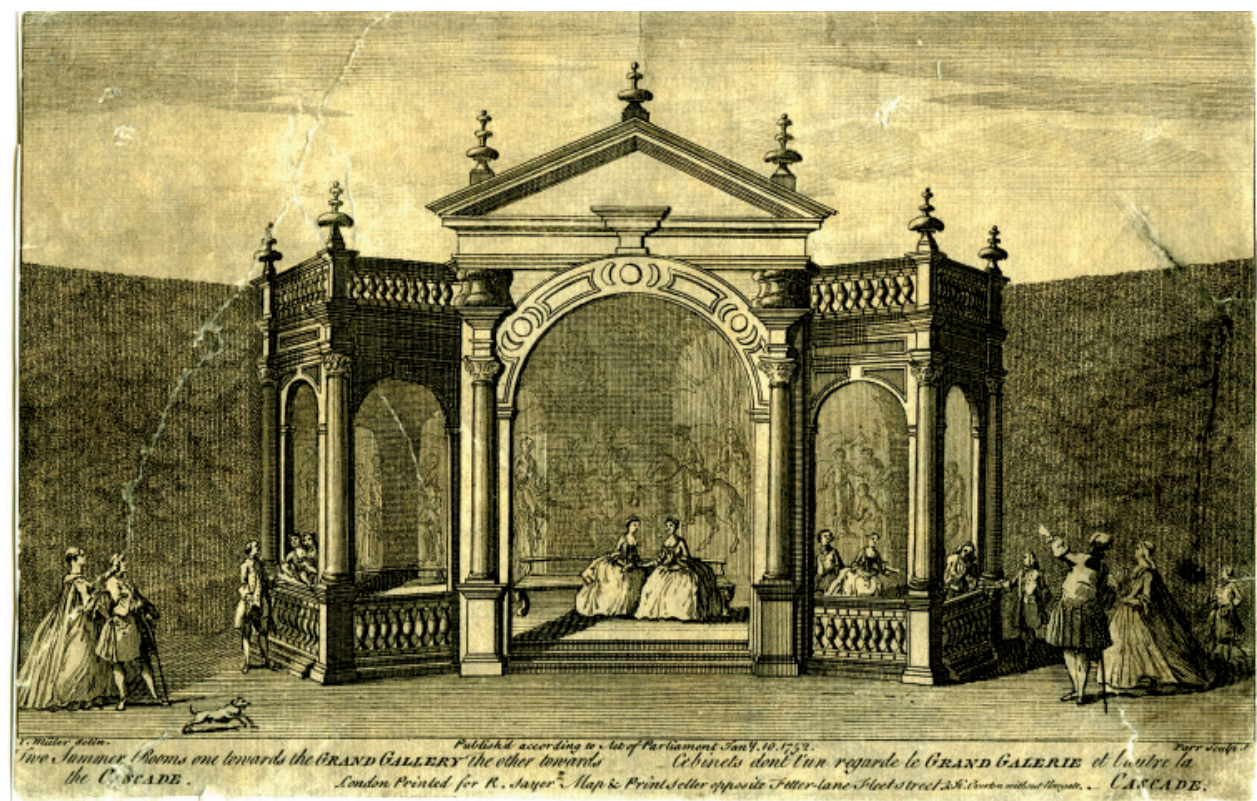

3 | Gartenkabinette in Herrenhausen, Kupferstich von Parr nach J. Müller, 1752 (Privatbestand des Autors). 
nehmung des alda von Hofchargen servirten Frühstücks, bestehend in einer mit kalten Speisen besetzten Tafel, nebst Theé, Caffé und Chocolade gangen, sondern haben sich nebst bey sich im Wagen habenden Printz Friedrich von Braunschweig nach einer kurtzen Verweil- und Unterredung mit dem Printzen Ernst von Mecklenburg-Strelitz, dem Herrn Hofmarschall v. Lichtenstein, der Frau Premier-Ministerin v. Münchhausen, und dem Herrn Feldmarschall v. Spörcken so fort wieder eingesetzet und sind, ohn etwas von den offerierten Frühstück zu nehmen, weiter $[\ldots]$ gefahren. $\ll^{11}$

Gleiches wiederholte sich kurz danach mit dessen Bruder, dem Prinzen Heinrich, der immerhin eine Tasse Kaffee zu sich nahm. Bei anderen Gelegenheiten setzte sich Heinrich jedoch mit dem hannoverschen Hof auch zu Tisch, um nicht unhöflich zu wirken.

Das Zeremonialbuch hielt bis Ende der 1720er Jahre nur dann Situationen im Freien fest, wenn diese mit Durch- oder Anreisen von Personen zu tun hatten. Um die Bedeutung der Residenz nicht herabzusetzen, wurden weder der Garten noch die Umspannstationen zu Orten des Zeremoniells erklärt, so dass dort auch Höflichkeitsbezeugungen reduziert, dem Gast freigestellt oder gar aufgehoben wurden. Zu Beginn des 18. Jahrhunderts scheint also noch eine deutliche Trennung zwischen privater und zeremonieller Sphäre zu bestehen: war der Innenraum dem Zeremoniell vorbehalten, so scheint der Außenraum (abgesehen von der Einholung der Botschafter) eine Privatheit zu bieten.

Diese Einstellung änderte sich jedoch, als ein wichtiger Teil des hannoverschen Hofes mit der Ernennung Georgs I. zum britischen König 1714 nach England ging und dort das englische Hofleben kennen lernte. Im Unterschied zu Hannover wurden zum ersten Mal Veranstaltungen des Hofes im Freien erwähnt, wie etwa am 1. August 1717, dem Tag der Proklamation Georgs I. zum englischen König, als in Hampton Court in Pavillons am Bowlinggreen Hof gehalten wurde. ${ }^{12}$

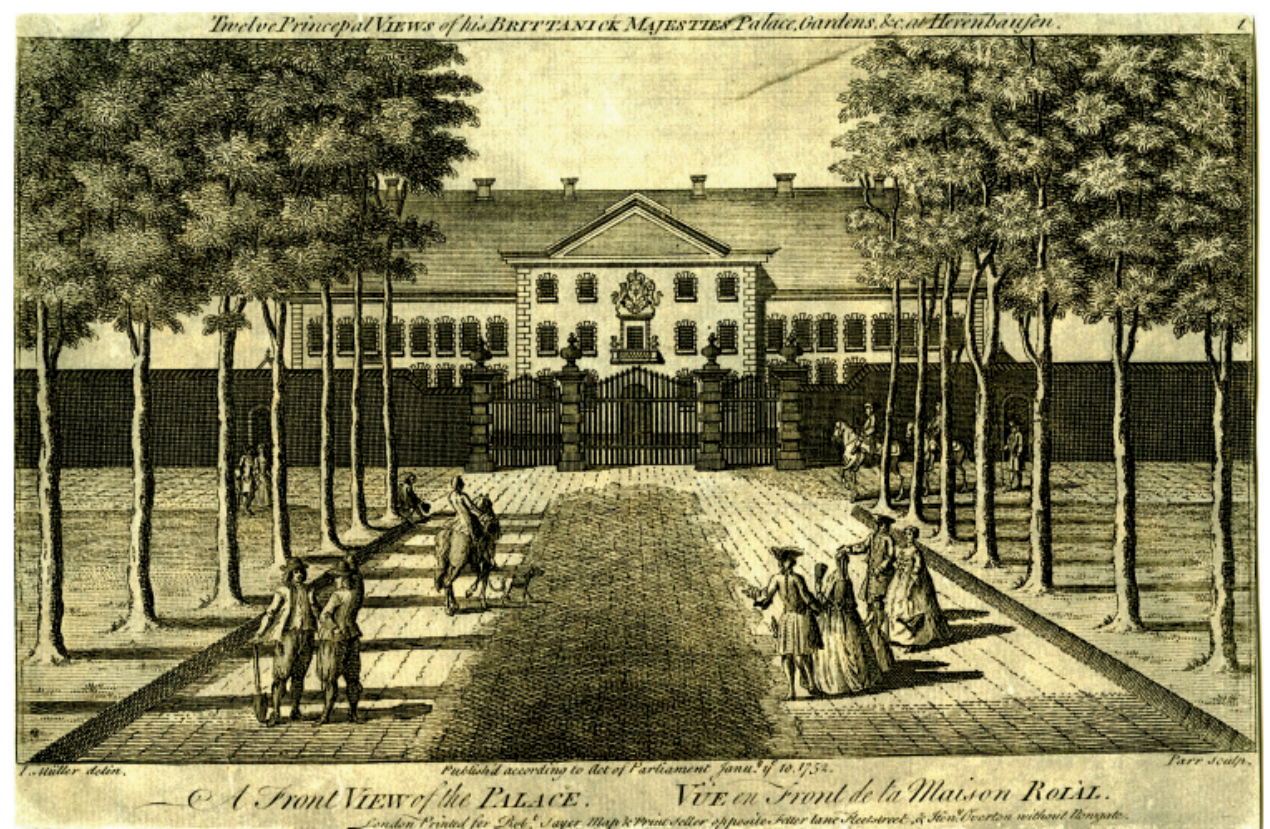

1 | Blick auf das Schloss Herrenhausen, Kupferstich von Parr nach J. Müller, 1752 (Privatbestand des Autors). 
Es folgten Berichte, dass der König das erste Mal Parforce-Jagden ritt und auf FeldhuhnJagd ging. ${ }^{13}$ Als sich der Monarch im Mai 1718 in Kensington befand, verzeichnet das Buch: „Das Abends wurde alle Compagnie von Distinction in den Garten gelassen, und in der Orangerie auch in der grünen Gallerie Ball gehalten. Um 11 Uhr war nechst dem Garthen im Park Ein Feuerwerck. ${ }^{14}$ Wenig später fand am Proklamationstag in der Orangerie ein weiterer Ball statt. Für 1721 wird festgehalten, daß sonntags der Kronprinz und seine Gemahlin nach Kensington zum Gottesdienst und zur Cour kamen. Einmal besuchte sie auch der König in Richmond, "um daß Haus und den Garten zu besehen«. Ein Jahr später berichtet man von einer militärischen Übung im Hyde Park, wobei man bei Lord Caddogan »im Park unterm Zelte« speiste. Ein paar Tage später, wieder am Proklamationstag, fand anstelle des wahrscheinlich als zu privat empfundenen Balls eine öffentliche Truppenparade statt: "Auch waren alle Boutiquen im Hide Parck illuminiert, und viele bonfeuer vor der fronte gemachet. $\aleph^{15}$

Das sich im Außenraum abspielende britische Zeremoniell mag u.a. auf die Notwendigkeit zurückzuführen sein, sich in einer konstitutionellen Monarchie dem Volk zu zeigen und dieses am höfischen Leben teilhaben zu lassen. Dies reichte vom prunkvollen Einholen der königlichen Jacht auf der Themse bis hin zu Händels später öffentlich aufgeführter Feuerwerks- und Wassermusik. Den klaren Grenzen zwischen Außen- und Innenraum, zwischen Garten und Palast, zwischen Privatsphäre und Zeremoniell, wie sie in Deutschland bestanden, scheint in England nicht die gleiche Bedeutung innegewohnt zu haben.

In Hannover, das durch seine ständisch ausgerichtete Gesellschaft ein anderes politisches System besaß, konnte zwar die Bedeutung nicht übernommen werden, doch scheint die Form der Repräsentation einen bleibenden Eindruck auf Georg I. hinterlassen zu haben. Die Möglichkeiten des zwangloseren Umgangs, die durch Veranstal- tungen im Freien gegeben waren, wurden zunehmend genutzt und vom Zeremoniell erfasst. So wird im Zeremonialbuch 1719 beim Besuch des Königs ebenso wie zuvor in Kensington ein Ball in der Herrenhauser Orangerie erwähnt, gefolgt von einer »Hühnerjagd «, zu der »ein Kochzelt, Ein Eßzelt und Ein Marschalls Eßzelt" in Isernhagen aufgebaut wurden. ${ }^{16}$ Im Folgejahr wird sogar verzeichnet, dass Georg I. und der zu Besuch weilende preußische König sowie Ernst August, Herzog von York, bei »Mylord Stanhope « und das andere Mal beim Hofmarschall von Bülow gegessen hätten. Obwohl das Protokoll noch vermerkt, dass Stanhope den Herrschaften an der Kutsche aufgewartet habe, bewegte man sich hier schon auf neuem Terrain: Man begab sich außerhalb des Schlosses zu Privatleuten, um hier "ohne Zeremoniell«, wie die Quellen häufig berichten, an einem Essen teilzunehmen. Plötzlich verzeichnet man nicht nur die Rückkehr des Königs aus Bad Pyrmont, sondern hält auch seinen privaten kleinen Fußmarsch fest, der ihn an die von einem englischen Mechaniker gerade errichtete Fontäne im Garten führte: Dieser »stiegen bei der Maschine aus, und kamen zu Fuß durch den Garten nach Herrenhausen. $\ll^{17}$

Ursprünglich von den englischen Geistlichen Benson und Holland zur Wasserversorgung der Stadt Shaftesbury erfunden, erkannte der König, welchen repräsentativen Charakter diese Technik in Deutschland haben würde. Er übernahm sie, belegte sie jedoch mit einer völlig neuen Bedeutung. ${ }^{18}$ Ähnlich wie schon zuvor auf dem Karlsberg in Kassel war sie als ein Symbol der Macht geplant. Dies zumindest legen auch die Eintragungen im Zeremonialbuch nahe, die sie als ein wichtiges Element der höfischen Repräsentation charakterisieren: Der König ordnete selber an, wann, wie hoch und mit wie vielen Rädern sie betrieben werden sollte. ${ }^{19}$ Am 30. Juli 1752, berichtet man sogar, »sprang die große Wasserkunst mitallen Rädern, wovon der Oberkammerh[err] die fremden Minister benachrichtigte. $\aleph^{20}$ Sie war damals die höchste mechanisch betriebene 
Gartenfontäne in Europa. Mit diesem technischen Wunder wurde der Garten eine höfische Attraktion und damit auch zu einem Ort des Zeremoniells: Als 1731 der Herzog von Lothringen kam, wird ausdrücklich vermerkt, dass dieser die Wassermaschine, die Fontäne, den Garten und die Galerie besichtigte, wobei ihn der Gartenintendant herumführte. 1738 kam Friedrich II., wenig später auch seine Schwester und 1744 Clemens August als Fürstbischof von Hildesheim. Bis ins ausgehende 18. Jahrhundert wurden diese Besuche im Zeremonialbuch verzeichnet.

Mit dem Regierungswechsel 1727 wurde Herrenhausen zur barocken Sommerresidenz ausgebaut und der Außenraum mehr und mehr genutzt, so dass sich erst in jener Zeit das von der Forschung evozierte festliche Bild zeigt. Aufschlussreich ist, dass Georg II. bei seinem ersten Besuch 1729 gleich nach seiner Ankunft nach Herrenhausen fuhr, um mit dem Gartenintendanten von Hardenberg das Schloss und den Garten zu besehen. Wenig später besuchte er sein neu erworbenes Schloss: »Dienstag, d. 7ten Juny. sind Sr. K. Majestet nach Mon Brillant gewesen und haben daselbst Hauß und Garten besehen. $«^{21}$

Über die Zeit von 1727 bis zum letzten Besuch Georgs II. im Jahr 1755 gab zuletzt Heike Palm Auskunft. ${ }^{22}$ Demnach besuchte er innerhalb seiner Regierungszeit zwölf Mal Hannover, wobei ein Schwerpunkt in den 1740er Jahren lag, als ein politisches Kräftemessen den europäischen Kontinent bestimmte. In dieser Zeit rückte auch das Herrenhäuser Galeriegebäude, das 1694 durch den Italiener Tomaso Giusti erbaut worden war, in den Mittelpunkt des höfischen Geschehens: Assembléen wurden dort gegeben, ebenso Komödien und Bälle. Anders als in den Jahren zuvor taucht damit auch der Gartenraum verstärkt im Zeremonialbuch auf.

Regelmäßig wurde nun das bereits 16891692 erbaute Heckentheater benutzt. Bei dessen erster Erwähnung wird berichtet, dass man wegen einsetzenden Regens das Stück in der Orangerie weiterspielte. ${ }^{23}$ Auch beim Besuch des schwedischen Königs 1731 wird vermerkt: "Da nun das Wetter zur Promenade nicht favorable war, so wurde Order gegeben, so gleich [das Essen] anzurichten." Das Risiko, sich dem Unbill des Wetters preiszugeben, ließ jedes Gartenfest zu einem Drahtseilakt werden. 1735 wurde dennoch das erste Fest im Großen Garten verzeichnet, in dessen Zentrum das Galeriegebäude und das Gartentheater standen:

»d. 18ten war Ball en Masque auf dem illuminierten Theatro im Garten, auf folgende Ahrt; Der Festin war in der Stadt an alle Persohnen vom 1ten und 2ten rang angesagt [...] Das Theatrum war solchergestalt illuminiert, daß um die Pyramiden Leisten Schlagen weise gezogen und mit Laub bewunden waren, und an den Bäumen Gläser mit Waßer und Oel befestiget wurden, unten am Theater und auf dem Fuß[?] der Fontaine, desgleichen auf den Treppen, waren Wachs Lampen, und oben auf den Pyramiden ein Licht mit einem Wind Glas gesetzt. Die Passagen nach dem Garten, so unnöthig, wurden verschlosen und die übrigen Pforten besetzt, welche ordre hatten niemand, als gute Masquen, in den Garten zu laßen. Die Gallerie ward gewöhnlich illuminiert, oben im Schloße cessirte die illumination. Im ersten Pavillon der Gallerie wurden die Cammern unten in der ersten Etage ausgeräumt, und benebst dem Ganzen illuminiert. [...] Die Buffets und SilbernTische standen in der Cammer rechter Hand in den Fenstern und waren mit Lichter erleuchtet, über dem Buffets aber war ein Spiegel bevestiget, welcher das point de vue vom Garten abgab [...]. « $^{24}$

Obwohl die Aufzeichnungen nahelegen, dass sich der Ball in der Galerie, in der man eine Kaffeebar aufgebaut hatte, fortsetzen würde, amüsierte man sich lieber im Garten. Man tanzte bis um halb vier Uhr morgens, wobei der König - wie das Zeremonialbuch 


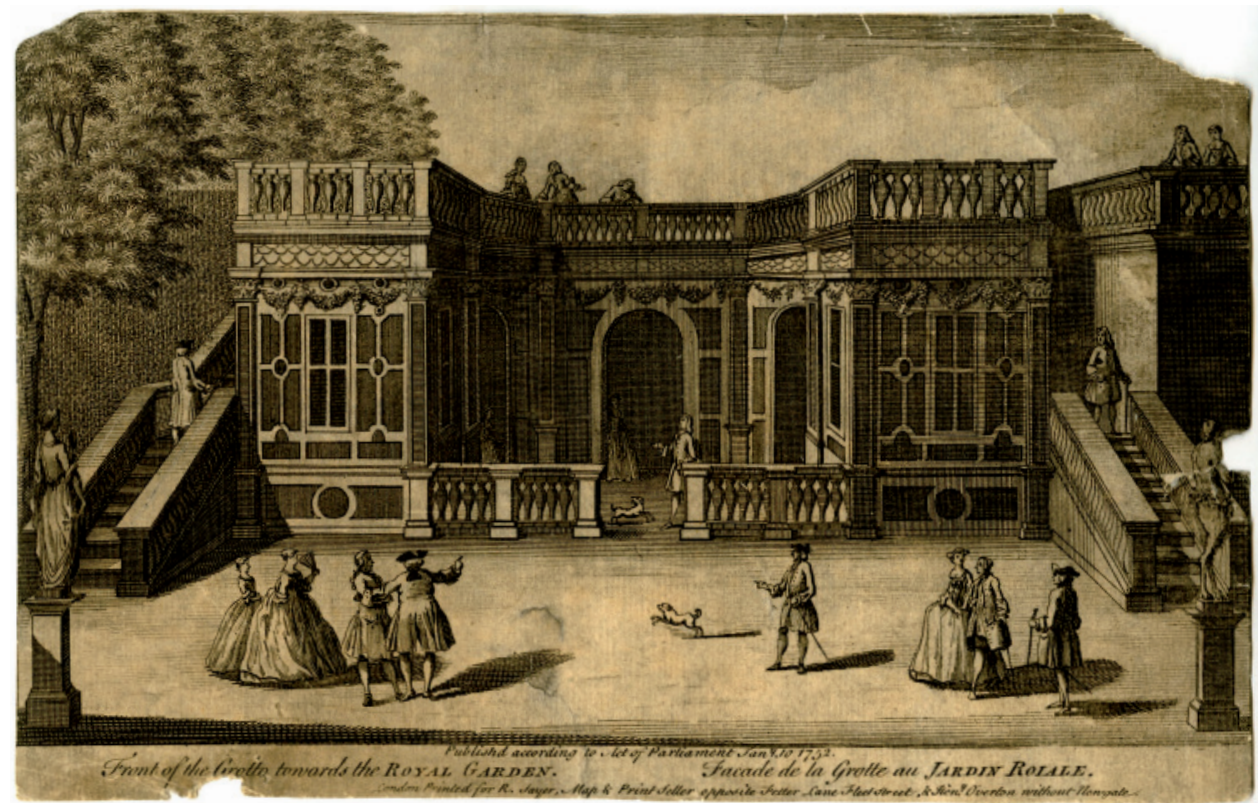

2 | Die Grotte in den Herrenhausener Gärten, Kupferstich nach S. Verne, 1752 (Privatbestand des Autors).

ausdrücklich vermerkt - bis zum Ende maskiert blieb. Am anderen Tag schrieb das Hofmarschallamt, dass der Monarch indisponiert sei. ${ }^{25}$

Diese Feste, bei denen 900-2000 Personen unterhalten wurden, waren Höhepunkte der Sommersaison. Selbst wenn der König in England war, gab man sie zu besonderen Anlässen, etwa dem Besuch des Kurfürsten und Fürstbischofs von Hildesheim Clemens August 1744:

"Nach der Tafel war Comedie im Garten und jedermann ward hinein gelassen, so daß etliche Tausend Menschen erschienen. Der Hof und andere vernehme Leute waren in größter Pracht, wie denn der König befohlen hatte, daß alles so seyn solle, als wenn er selber zugegen wäre. $\aleph^{26}$

Zuweilen fanden auch Feuerwerke in der Leineaue statt, die der König aus dem Garten verfolgen konnte. ${ }^{27}$

Die erste im Garten gehaltene Tafel verzeichnet das Zeremonialbuch erst 1748 . Erstaunlicherweise wurde diese zwar im kleinen Rahmen, jedoch mit großem Aufwand betrieben: "Abends speiseten Sr. Königl. Maj.tät im Garten gegen der Cascade über da dann das Buffet und Silber Tisch an der Seiten der Cascade gesetzet würden [...].«Am 19. und 20. Juni 1750 wird gleiches berichtet. ${ }^{28}$

Auch außerhalb des Gartens fanden Veranstaltungen statt, die das Zeremonialbuch festhält: War der gesamte Hof bei Prunkjagden und Truppenparaden zugegen, wurden Zelte aufgebaut. ${ }^{29}$ Auffällig ist, dass man mehr und mehr davon anschaffte (Tafelzelte, Garderobenzelte, Kochzelte), so dass man schließlich insgesamt elf davon besaß Der »Caffetier « Hero schenkte üblicherweise Tee, Schokolade und Kaffee aus. Später kam noch ein Konditor-Zelt mit Limonaden-Ausschank und Gebäck hinzu. Die ursprünglichen Feldstühle schienen dabei zunehmend von regulären Sitzmöbeln und Tischen abgelöst worden zu sein. Vom Grafen von Schaumburg-Lippe wird 1733 berichtet, dass auch er den König auf einer seiner Reisen in Zelten bewirten wollte, dieser jedoch nur auf eine Viertelstunde zu einer Unterredung 
blieb und dann weiterfuhr..$^{30}$

Neben den eigentlichen höfischen Veranstaltungen tauchen zunehmend bürgerliche Feste im Zeremonialbuch auf. Eine dieser Belustigungen war das Bürgerschießen, das neben dem Garten in Herrenhausen stattfand. Man berichtet 1732 sogar von begrünten Lauben, die die Regimenter zum Maibierfest hinter dem Großen Garten zum Tanzen und Bierausschank aufbauten. Mit einer Parade zog man durch die Herrenhäuser Allee, um dem König im Innenhof des Schlosses eine Referenz zu erweisen. Dieser revanchierte sich und besuchte den Festplatz, sah den Tanzenden im ObristenZelt $\mathrm{zu}$, verabschiedete sich aber bald, weil das Wetter schlecht war. Am Abend gingen die Feiernden durch den Garten und über den Schlossplatz wieder in die Stadt. 1735 erfolgte das gleiche Schauspiel.

Alle größeren Festlichkeiten, wie beispielsweise das Krönungsfest am 21. Oktober 1727 , wurden mit Feuerwerken und Illumination gefeiert. Hierzu beleuchteten die Einwohner Hannovers die Fenster ihrer Häuser, wobei das Zeremonialbuch die Strecke und den Aufwand, den einzelne Adlige und Bürger betrieben, hervorhebt. Über den anwesenden Kronprinzen wird berichtet, dass dieser inkognito aus einer kleinen Kutsche den Schmuck betrachtete und sogar zu Fuß die Stadt erkundete. Im Allgemeinen schien Georg gerne Spazieren zu fahren und sich Neuigkeiten anzusehen. Redecker berichtet beispielsweise von der abenteuerlich anmutenden Einfahrt des Hofes in einen Stollen im Oberharz. ${ }^{31}$ Bequemer fiel der Besuch des Königs im Weyheschen Garten aus, »um das darauf gebauete neue Haus zu besehen «, das seiner Mätresse, der Gräfin Yarmouth, gehörte.

Die Zeit nach dem letzten Besuch Georgs II. im Jahr 1755 hinterließ zunächst eine Agonie, die durch die Folgen des Siebenjährigen Krieges noch verstärkt wurde. Der Ball und die Illumination, die anlässlich des Besuches des Braunschweiger Erbprinzenpaares Karl Wilhelm Ferdinand und Augusta, Enkelin Georgs II., 1765 gegeben wurde, waren die letzten »barocken Feste«, die in Herrenhausen gefeiert wurden. ${ }^{32}$ Bereits Mitte der 1750er Jahre wusste der hannoversche Gartenintendant Friedrich Karl von Hardenberg, dass die Zeiten der barocken Gartentheater und Parterres vorüber waren, und unterbreitete Vorschläge für die Umarbeitung des alten formalen in einen landschaftlichen Garten. ${ }^{33}$

Der nächste Schritt wurde durch die Thronbesteigung Georgs III. getan, der seine hannoverschen Erblande nie besuchte. Herzog Carl und Ernst von MecklenburgStrelitz übernahmen als Schwäger des englischen Königs repräsentative Rollen. Von einer Hofhaltung kann man erst wieder 1786 sprechen, als Georgs zweitgeborener Sohn, Herzog Friedrich August von York und Bischof von Osnabrück, Statthalter in Hannover wurde.

Trotz Abwesenheit des Regenten bestand die Aufgabe darin, den Hof so zu repräsentieren, dass der hannoversche Adel aber auch gelegentliche fürstliche Gäste unterhalten werden konnten. In der Regel logierten die Gäste nicht in der Residenz, da man das dort zu erwartende Zeremoniell seitens des fürstlichen Hauses in Abwesenheit nicht zu erfüllen gedachte. Sie wohnten somit im weitaus bequemeren Herrenhausen oder dem Lustschloss Montbrillant, das als eine Art Gartenschloss fungierte. Das Zeremonialbuch berichtet 1788 , dass man den Garten und das Haus für fürstliche Besucher erleuchtete. ${ }^{34}$ Mit den Aufenthalten der königlichen Prinzen, die alle in England erzogen wurden, weichte das Zeremoniell jedoch vollkommen auf. Das Zeremonialbuch wird nunmehr zu einem Hoftagebuch, das von den Besuchen der Prinzen beim hannoverschen Adel auf dem Land berichtet, ihren Fahrten zum Tanz auf den Rehburger Brunnen oder von einem Mittagessen im Garten des illegitimen Großonkels, des Grafen von Wallmoden. Zuweilen ließ man sich einladen oder fuhr mit Gästen in die London-Schänke, die später nach dem berühmten Londoner Ver- 
gnügungspark »Vauxhall « benannt wurde. ${ }^{35}$ Diese Vermischung von Bürgern und Adligen führte schließlich auch zur Öffnung des einstmals privaten Großen Gartens in Herrenhausen für die Öffentlichkeit.

Indem man mehr und mehr Aktivitäten aus dem Innenraum der Residenzen in den Außenraum verlagerte, nahm auch das Zeremoniell ab. Man umging es gezielt. Der Blick nach England bestätigt dies: Georg III. unterwarf sich mit seiner Familie nur an den offiziellen Cour-Tagen und zu speziellen Staatsakten dem Zeremoniell. Die meiste Zeit verbrachte er als Privatmann, als "Farmer George", in Windsor oder in Kew Gardens, seinem Park, der damals noch nicht der Öffentlichkeit zugänglich war. Ähnlich wie seine Vorfahrin Sophie nutzte auch er den Garten als eine private Rückzugsmöglichkeit, fernab jeglichen Zeremoniells. Dies berichtet auch einer der letzten Einträge des Zeremonialbuches 1823: „Auf den 22ten May war eine Collation im Königlichen Thiergarten bey Kirchrode angesagt, wozu außer Ihro Königlichen Hoheiten auch des Herzogs und der Herzogin von Cambridge, der Prinzessin Augusta Königliche Hoheit [...]" sowie andere Gäste aus dem Adel anwesend waren. Wegen des einsetzenden Regens musste jedoch alles in den Wallmodenschen Garten verlegt werden.

»Man versammelte sich um 6 Uhr ohne Ceremoniel. [sic!] Das Musik Corps der Husaren Garde war im Garten neben den gesellschafts Zimmern postirt. In den Zimmern selbst wurde gespielt und Thee, Lymonade, Punsch, Eis und Backwerk präsentirt. $\ll^{36}$

Ist der Garten also das genaue Gegenteil vom Zeremoniell? Hartmann und Ottersbach beantworten diese Frage folgendermaßen:

»Der Garten ist ein Teil des Schauplatzes, auf dem sich das höfische Leben abspielt. Doch ist er im Gegensatz zur Residenz ein beinahe zeremoniell-freier Bereich.
Es gab kein spezielles Zeremoniell für den Gartenraum. Das Zeremoniell spielte nur dann im Garten eine große Rolle, wenn dort Feste und ähnliche Divertissements stattfanden. Aber auch innerhalb des Gartenraumes dürfte es bestimmte Regeln gegeben haben, welche sich allerdings in einer entsprechenden Literatur nicht greifen lassen. «37

Ging man also bislang davon aus, dass der Garten durch seine Kabinette und Galerien (Berceaux) die Architektur des Schlosses widerspiegelt, so muss er doch anders, möglicherweise sogar als Gegenentwurf dazu funktioniert haben. Er eröffnete die Möglichkeit, sich freier zu bewegen und bot damit auch wiederum neue Räume für Festlichkeiten.

Die Eintragungen im hannoverschen Zeremonialbuch belegen, dass der frühbarocke Garten zunächst nicht in den Bereich des Zeremoniellen, sondern des Privaten gehörte. ${ }^{3}$ Erst das Vorbild der anderen durch Standeserhöhungen aufgewerteten Höfe, wie Berlin und Dresden, die Erlangung der englischen Königskrone, aber auch das im zunehmenden Maße umgesetzte Vorbild Versailles, führten neben einer Steigerung des Repräsentationsbedürfnisses zur Entdeckung von Alternativen, die vor allem der Gartenraum bot. ${ }^{39}$ Der fließende Übergang zwischen Ungezwungenheit und Zeremoniell eröffnete Möglichkeiten für Überraschung und Abwechslung, die dem üblichen Hofzeremoniell nicht unbedingt innewohnten. Die vermehrten Eintragungen im Zeremonialbuch unter Georg I. und Georg II. weisen darauf hin, dass man den Spielraum, den der Garten bot, gezielt nutzte und einsetzte. $^{40}$

Obwohl sich der kulturelle Wandel in der zweiten Hälfte des 18. Jahrhunderts, der u.a. zur Entstehung des Landschaftsgartens und zu einer »Privatisierung « des Hofes führte, nachhaltig auf den Inhalt des hannoverschen Zeremonialbuches (nunmehr: "Hoftagebuch «) auswirkte, veränderte sich die Funktion des Außenraumes kaum. Trotz des 
Wandels der Gartenstile und damit auch der kulturgeschichtlichen Hintergründe, bleibt die Funktion des höfischen Gartens unverändert, ist er doch noch immer Idylle, Paradies und Erholungsraum. Um dies jedoch genauer zu untersuchen, muss sich die bisherige Forschung noch intensiver neuen Möglichkeiten öffnen, wie beispielsweise der Sozialgeschichte des barocken Gartens. ${ }^{41}$
1 Alvensleben, Udo von: Herrenhausen. Die Sommerresidenz der Welfen, Berlin 1929; zur 300-JahrFeier entstand der Katalog: Meyer, Karl H.: 300 Jahre Herrenhausen, Hannover 1966, gefolgt von populären Titeln wie: Troll, Hans J.: Schönes Herrenhausen, o.O. 1966; Morawitz, Kurt (Hg.): Festliches Herrenhausen, Hannover 1977, Ders.: Glanzvolles Herrenhausen, Hannover 1981.

2 Über den Hof in Hannover und Großbritannien siehe: Richter-Uhlig, Uta: Hof und Politik unter den Bedingungen der Personalunion zwischen Hannover und England, Hannover 1992, hier vor allem S. 25-33: Hofleben in Hannover; Patze, Hans: Zwischen London und Hannover. Bemerkungen zum Hofleben in Hannover während des 18. Jahrhunderts, in: Berglar, P.: Festschrift für Hans Tümmler zu seinem 7o. Geburtstag, Köln/ Wien 1977, S. 95-123 (u.a. in Bezug zu den Zeremonialbüchern). Erst in letzter Zeit sind durch Heike Palm, Hubert Rettich und Bernd Adam Grundlagen und damit ein objektiveres Bild des Großen Gartens geschaffen worden. Literatur und Forschungsstand siehe: Palm, Heike: Rettete eine Dissertation den Großen Garten zu Hannover-Herrenhausen?, in: Blanke, Harald (Hg.): Ein brüderliches AlllianceEuvre. Beiträge zur Gartenkunst, Geschichte und Denkmalpflege im Werk Udo von Alvensleben und Anco Wigboldus, Hundisburg 2004, S. 55-77. Dies.: Der Fürst auf der Gartenbühne und die Arbeit hinter den Kulissen. Nutzung, Pflege und Unterhaltung des Großen Gartens in Hannover-Herrenhausen in der ersten Hälfte des 18. Jahrhunderts, in: Lesemann, Silke/ Stieglitz, Annette von (Hg.): Stand und Repräsentation, Bielefeld 2004, S. 41-81. Adam, Bernd: Neue Funde zum barocken Ausbau der Schlossanlage in Hannover-Herrenhausen, in: Niederdeutsche Beiträge zur Kunstgeschichte 40 (2001), München Berlin, S. 59-97. König, Marieanne von (Hg.): Herrenhausen. Die Königlichen Gärten in Hannover, Göttingen 2006. Eine Zusammenstellung liefert: Stieglitz, Annette von: Höfisch-barocke Feste in Herrenhausen, in: Schmidt, Hans-Dieter (Hg.): Feste und Feiern in Hannover. Hannoversche
Schriften zur Regional- und Lokalgeschichte, Nr. 10, Bielefeld 1995, S. 85-111. Bredekamp, Horst: Leibniz und die Revolution der Gartenkunst, Berlin 2012.

3 Wallbrecht, Rosemarie: Das Theater des Barockzeitalters an den welfischen Höfen Hannover und Celle, Hildesheim 1974.

4 Wiewelhove, Hildegard (Hg.): Gartenfeste. Das Fest im Garten - Gartenmotive im Fest, Bielefeld 2000. Differenzierter: Frühsorge, Gotthardt: Theater, Feste, Maskeraden, in: König, Marieanne von (Hg.): Herrenhausen. Die Königlichen Gärten in Hannover, Göttingen 2006, S. 79-94.

5 Das fünf Bände umfassende Zeremonialbuch ist in Ausschnitten von Carl Ernst von Malortie publiziert worden: Beiträge zur Geschichte des BraunschweigLüneburgischen Hauses und Hofes, Hannover 1860-1884, 7 Hefte. Es befindet sich als Depositum 103 IV Nr. 321 (1680-1713), 323 (1714-1723), Nr. 324 (1734-1774), Nr. 325 (1775-1825) im Niedersächsischen Landesarchiv Hannover (NLA HA), später wird das Buch als Hofjournal weitergeführt (bis Dep. 103 Nr. 354, 1866). Die Seiten sind alt-paginiert, jedoch chronologisch aufgebaut. Für die Möglichkeit der Einsichtnahme sei S.K.H. dem Prinzen von Hannover gedankt.

6 Das entsprechende Reglement wurde 1707 verfasst: Malortie (s. Anm. 5), 1. Heft, 186o, S. 3-5.

7 Teilweise wurden die Botschafter auch zur Tafel geladen, was jedoch nicht immer angenommen wurde. In der Regel folgte der Audienz ein Besuch des Kronprinzenpaares im Stadtschloss, so dass die Allee ein zweites Mal durchfahren werden musste.

8 Malortie, Carl Ernst von: Der Hannoversche Hof unter dem Kurfürsten Ernst August und der Kurfürstin Sophie, Hannover 1847, S. 79f. Das Zeremonialbuch geht nicht auf das Ballett ein, welches ihr zu Ehren im Garten aufgeführt worden sein soll. Vgl. dazu: Wallbrecht 1974, S. 77 f.

9 Schnath, Georg: Geschichte Hannovers im Zeitalter der neunten Kur und der englischen Sukzession 1674-1714, Bd. 2, Hannover 1976, S. 311-315. Der Zar sollte im „Schloss“ logieren. Als er jedoch die vielen Menschen auf dem Wall dort sah, die er 


\section{Naturraum Und ZERemoniell}

ausdrücklich nicht gewünscht hatte, nahm er Quartier im Rathaus und wollte dort auch bleiben. Der hannoversche Hofmarschall überredete ihn darauf mit einer List: Der russische Gesandte wurde in die Kutsche des Zaren gesetzt, so dass der Herrscher mit seinem Vetter und einem Dolmetscher zu Fuß unerkannt von der Volksmasse das Schloss betreten konnte. Der anschließende Abend verlief im kleinsten Kreis ohne jegliches Zeremoniell (s. Aufzeichnungen vom 25. bis 28.7.1697).

10 Aus Redeckers Aufzeichnungen über die Jahre 1692-1721, in: Hannoversche Geschichtsblätter 11 (1908), S. 355.

11 NLA HA Dep. 103 IV Nr. 324, weitere Besuche: 11.8.1738 Kronprinz Friedrich (wegen Fontäne); Juni 1763 Friedrich II.; Juni 1768 Prinz Heinrich: auf dem Weg nach Het Loo stieg er in der LondonSchänke ab, hielt Tafel in der Herrenhauser Galerie und besah mit der ganzen Gesellschaft die Fontäne; Dep. 103 IV Nr. 325, 2.7.1781 Prinz Heinrich zum Abendessen in Montbrillant, Mitternacht Abreise nach Spa; am 4.9.1781 Rückreise mit kurzer Pause in Montbrillant.

12 Original: „in einem von die Außenhäuser am Boulinggreen"; möglicherweise handelt es sich hier um das Banqueting House (NLA HA Dep. 103 IV Nr. 323, 1.8.1717). Das deutsche Zeremonialbuch wird in England übergangsweise weiter geführt.

13 Entgegen der Annahme, dass die Parforce-Jagd aus Frankreich übernommen wurde (obwohl die Großtante Liselotte von der Pfalz sie bereits ritt), lernte sie Georg I. erst in England kennen und muss sofort begeistert gewesen sein, s. Einträge 19., 23., 26., 29.8. 1717, NLA HA Dep. 103 IV Nr. 323.

14 NLA HA Dep. 103 IV Nr. 323, 20.5.1718.

15 Ebd., 1.8.1722.

16 Ebd., 31.8.1719.

17 Vgl. auch NLA HA Dep. 103 IV Nr. 323, Juni/Juli 1720; s.a. Nr. 324, Dez. 1726 von Kensington nach St. James's gefahren „und im Parck vor der Garten Thür ausgestiegen“. Das Zeremonialtagebuch vermerkt in jenen Jahren noch kursorisch Geschehnisse am Londoner Hof.

18 Adam 2001, S. 8of.

19 Lampe, Joachim: Aristokratie, Hofadel und Staatspatriziat in Kurhannover, Göttingen 1963, Bd. 1, S. 119.

20 Spilcker, Burchard Christian von: Ueber des Königs Georg II. Reisen nach Hannover von 1744-1755. Aus einem, vom Oberkammerherrn Grafen von Bülow geführten Tagebuche, in: Spiel, G.H. (Hg.): Vaterländisches Archiv oder Beiträge zur allseitigen Kenntniß des Königreichs Hannover, 1. Bd., Hannover 1821 , S. 289.

21 NLA HA Dep. 103 IV Nr. 323, 7.6.1727.

22 Palm 2004, S. 43.

23 Wallbrecht 1974, S. 77 erwähnt neben dem Theaterspiel vom 26.6.1681 auch 1687/88 eine Theaterlaube. Stieglitz 1995, S. 105, geht von Theateraufführungen unter Herzog Johann Friedrich (gest. 1679) in Herrenhausen aus.
24 NLA HA Dep. 103 IV Nr. 324, 18.6. 1735.

25 Ebd.

26 Redecker (Anm. 10), S. 240.

27 s.a. Wallbrecht 1974, S. 115-123.

28 NLA HA Dep. 103 IV Nr. 324, 28.6.1748. Hierauf geht schon Zacharias Conrad von Uffenbach bei seiner Reise ein 1710: Merckwürdige Reisen durch Niedersachsen, Holland und Engelland, Frankfurt / Leipzig 1753, S. 417. Anstelle der ehem. Grotte „sind kleine Cabinetgen um darinnen im Sommer zu speisen, gemacht". Vgl. Kupferstich J.J. Müllers und Jost van Sasses auf einer Spielkassette im Historischen Museum Hannover VM 35295.

29 Vgl. hierzu das Gemälde „Die Truppenparade bei Bemerode“, 1725, Historisches Museum Hannover. Siehe auch: Richter-Uhlig 1992, S. 32, Fußnote 69.

30 Aus Redeckers Aufzeichnungen über die Jahre 17241762, in: Hannoversche Geschichtsblätter 12 (1909), S. 179-256, hier S. 214.

31 Redecker (Anm. 30), S. 204 (25.7.1729).

32 Malortie (Anm. 5), 2. Heft, S. 57-6o.

33 Köhler, Marcus: Friedrich Karl von Hardenberg's (1696-1763) Journeys to England and his Contribution to the Introduction of the English Landscape Garden to Germany, in: Garden History 1998 (25/2), S. 212-217.

34 Patze 1997, S. 121-125. Über den Besuch Friedrich Wilhelms II. von Preußen, der am 2.6.1788 die Wasserspiele, den Großen Garten und die Orangerie besichtigte.

35 Ähnliche Vergnügen außerhalb des Hofbezirks muss es auch schon vorher (1693) gegeben haben; sie erscheinen jedoch nicht im Zeremonialbuch, vgl. Schnath, Georg: Der Königsmarck-Briefwechsel, Hildesheim 1952, S. 154.

36 NLA HA Dep. 103 IV Nr. 325, 22.5.1823.

37 Hartmann, Christin / Ottersbach, Christian: Der höfische Garten, in: Bender, Eva / Berns, Jörg Jochen (Hg.): Erdengötter - Fürst und Hofstaat in der frühen Neuzeit, Marburg 1997, S. 215f.

38 In diesem Zusammenhang muss auch die Theateraufführung von 1681 für Sophie Amalie im Garten gesehen werden, vgl. Wallbrecht 1974, S. 77f.

39 Hingewiesen sei auf Gartenfeste wie z.B. die 1702 belegte Theateraufführung und Seeschlacht im Charlottenburger Garten oder 1700 die Vermählungsfeier Luise von Brandenburgs mit dem Kurprinzen Friedrich von Hessen-Kassel in Oranienburg. Zu Ludwig XIV.: Spagnolo-Stiff, Anne: Barocke Gartenfeste und ihr Prototyp, die Versailler 'Divertissements', in: Wiewelhofe 2000, S. 35-39.

40 z.B. Malortie (Anm. 5), 1. Heft, Hannover 1860, S. 57: „Georg I. war in dem Augenblick in seinem Orangeriegarten beschäftigt, Blumen zu cultiviren, als jene Gesandtschaft eintraf und empfing dieselbe in diesem Garten, der dadurch ein besonderes Interesse erhält."

41 Gebhard, Walter (Hg.): Sozialgeschichtliche Aspekte des Gartens. Gardens in the social history, Frankfurt 2002. 